\title{
A dissociation between detection and identification of phobic stimuli: Unconscious perception?
}

\author{
Paul Siegel, Edward Han, Don Cohen, and Jason Anderson \\ Department of Behavioral and Social Sciences, Psychology, SUNY/Westchester and Purchase \\ College, Westchester, NY, USA
}

\begin{abstract}
A psychophysical paradigm for investigating unconscious perception was used to test the hypothesis of dissociation between detection and identification of phobic stimuli. Spider-phobic and non-phobic participants were presented with masked images of spiders and flowers and an equal number of control stimuli in a random sequence. After each masked stimulus was flashed, participants first reported whether or not an object was presented. Then they identified each stimulus as either a spider or a flower, regardless of their prior detection response. Phobic participants identified both detected and undetected spiders better than chance, as assessed by two measures of response bias. They did not exhibit dissociation between detection and identification for flowers. Non-phobic participants did not exhibit detection-identification dissociation for either spiders or flowers. These results are consistent with the interpretation that phobic individuals unconsciously perceive their feared stimulus, and constitute the first direct demonstration of such for emotional stimuli.
\end{abstract}

Keywords: Detection-identification dissociation; Unconscious perception; Phobia.

A basic proposition of prevailing theories of fear (LeDoux, 1996; Öhman \& Mineka, 2001) and anxiety disorders (Barlow, 2002; Eysenck, 1992; Mathews \& Mackintosh, 1998; Mogg \& Bradley, 1998; Williams, Watts, MacLeod, \& Mathews, 1997 ) is that perception of threatening stimuli occurs without awareness. There is ample evidence to support this claim. Many studies have found dissociations between awareness of and responses to fear-relevant and phobic stimuli, as evidenced by a variety of behavioural, psychophysiological and neurobiological indices. For example, a rich econ- omy of neuroimaging studies has demonstrated amygdala activation by masked, threatening stimuli (Morris, Öhman, \& Dolan, 1998, 1999; Phelps, 2005; Whalen et al., 2004; Williams, Morris, McGlone, Abbott, \& Mattingley, 2004). Such activation by masked stimuli appears to be lateralised and recruit different neural circuits than do unmasked, consciously processed stimuli (Carlsson et al., 2004; Morris et al., 1998), suggesting a dual neural architecture of fear based on awareness of stimuli. This body of research provides a biological basis for Öhman and Mineka's (2001) theory of the

Correspondence should be addressed to: Paul Siegel, School of Natural and Social Sciences, Purchase College/SUNY, 735 Anderson Hill Road, Purchase NY, 10577, USA. E-mail: paul.siegel@purchase.edu

This article was originally published with errors. This version has been amended. Please see Corrigendum (http://dx.doi.org/10. 1080/02699931.2013.825509). 
fear module: a neural system for automatically processing stimuli that posed threats in the evolutionary history of mammals. Öhman and colleagues have used masking procedures to show consistently that autonomic responses can be activated by and conditioned to masked, fear-relevant stimuli (Esteves, Dimberg, \& Öhman, 1994; Katkin, Wiens, \& Öhman, 2001; Öhman \& Soares, 1993, 1994, 1998). For example, such stimuli activated equivalent or even stronger galvanic skin responses than unmasked stimuli in fearful participants (Öhman \& Soares, 1994). Thus, the fear module is believed to primarily involve non-conscious processes that are relatively impenetrable to conscious, cognitive control: “... in fear, unconscious processing might dominate conscious processing”.

Despite such empirical and theoretical convergence on the non-conscious basis of fear, disagreement remains as to whether unconscious perception of threatening stimuli has been adequately demonstrated (Holender \& Duscherer, 2004; Pessoa \& Adolphs, 2010). Awareness measures have had a variety of limitations that have rendered them either not exhaustive of or exclusive to conscious processes (see Lovibond \& Shanks, 2002, for a review). Most of these limitations boil down to the problem of establishing a threshold for null stimulus detection, which may be impossible to do (Macmillan, 1986). Furthermore, unconscious perception has only been indirectly demonstrated by dissociations between awareness of and behavioural response to masked stimuli. By definition, perception without awareness can only be directly demonstrated by dissociations between awareness and perception of stimuli. Only three studies have tested a detection bias for masked, threatening stimuli (Becker \& Rink, 2004; Windmann \& Kruger, 1998; Winton, Clark, \& Edelmann, 1995). All three found that phobics were no more likely to detect their feared stimulus than non-phobics.

This study provided a more specific, rigorous test of unconscious perception of threatening stimuli in a few ways. It was based on a paradigm for assessing awareness-perception dissociations that addresses the null stimulus detection problem. Previously, the paradigm had only been used with non-affective stimuli. Whereas the three aforementioned studies solely assessed a detection bias by comparing detection of masked phobic and non-phobic stimuli in separate blocks (each relative to control stimuli), this study provided a more exhaustive test of a perception bias by comparing detection and identification of these stimuli within the same block. Finally, this study built on prior stimulus detection measures by combining the validity-enhancing features of subjective and objective measures of awareness.

In the detection-identification dissociation (DID) paradigm, stimulus detection is compared to an alternative index of perception that is believed to be sensitive to unconscious processes (Macmillan \& Creelman, 1991; Marcel, 1983; Weiskrantz, Warrington, Sanders, \& Marshall, 1974). From the vantage point of the DID paradigm, a perceptual bias is most likely to be evidenced not by stimulus detection, but concurrent measurement of stimulus detection and identification. For example, in a series of experiments conducted by Merikle and Reingold (1990), participants reported if they detected letter strings, and then identified them as either words or non-words. When letter strings were presented that they were able to detect, they identified both words and nonwords better than chance. When letter strings were presented that they were unable to detect, they only identified words better than chance, not nonwords. Thus, there was dissociation between detection and identification of words, but not non-words. This was interpreted to mean that words were perceived without awareness. The advantage of the DID paradigm is that stimulus detection is validated as a measure of awareness by showing that a variation in detection (detected and undetected stimuli) is associated with qualitative differences in perception, rather than by trying to establish an awareness threshold. Because the effects of undetected stimuli would generally be expected to be weaker than those of detected stimuli, if effects associated with these purportedly unaware and aware states differ from this expected pattern-e.g., letter strings are identified as words whether or not they are detected, whereas letterstrings are identified as non-words only when they

\section{4}

COGNITION AND EMOTION, 201327 (7) 
are detected - it suggests that undetected stimuli were indeed perceived without awareness (Merikle \& Daneman, 2000; Wiens, 2006). Merikle and Reingold (1990) concluded that only familiar stimuli represented in memory would generate such dissociation between stimulus detection and identification.

This study adapted the DID paradigm to affective stimuli by comparing the detection and identification responses of spider-phobic and nonphobic participants to masked images of spiders, flowers, and unfamiliar, control stimuli. Based on sample evidence of unconscious responses to phobic stimuli, the hypothesis was that such stimuli would yield dissociation between stimulus detection and identification. That is, we predicted that spider-phobics would identify spiders better than chance whether or not they detected them, whereas non-phobics would identify spiders only when they detected them. If detectionidentification dissociations are bound by stimulus familiarity, however, they should be generated by both fear-relevant and fear-irrelevant stimuli (i.e., spiders and flowers), regardless of the participants' phobic status.

Three aspects of this study provided a more specific, rigorous test of unconscious perception of threatening stimuli. First, stimulus detection and identification stimuli were measured concurrently(Haase, Theios, \& Jenison, 1999; Merikle \& Reingold, 1990). On each trial, participants first reported whether or not a stimulus was presented - a detection response. Then they gave a second response, identifying the stimulus as either a spider or a flower-whether or not they previously reported detection of a stimulus. Concurrent measurement of stimulus detection and identification enables testing of the perception-dissociation hypothesis by directly comparing identification of detection "bits" and "misses": when a target stimulus is presented and detected, and when it is presented but not detected, respectively. Dissociation occurs if a target category is identified better than chance after detection misses as well as hits, rather than only after hits - i.e., whether or not a stimulus is detected, rather than only when it is detected.
Second, phobic and non-phobic stimuli were all presented in the same rather than in separate blocks stimuli (e.g., Becker \& Rinck, 2004). Directly comparing detection and identification of these stimuli within the same block made it possible to test in a single experiment whether stimulus recognition in the absence of detection is limited to familiar (flowers) versus fear-relevant (spiders for non-phobics) versus phobic (spiders for spider-phobics) stimuli. To focus on unconscious processing, the target stimuli were masked and presented at a very brief stimulus-onset asynchrony (SOA) between target and mask.

Third, following Wiens' (2006) recommendation, we built on prior stimulus detection measures by combining the validity-enhancing features of subjective and objective measures of awareness. On the one hand, measures of awareness should capture its inherently subjective nature (Bowers, 1984; Merikle, 1992). As Wiens (2006, p. 11) put it, "Because awareness is a process that is closer to 'noticing' than 'discriminating', a valid measure of awareness ought to capture what participants notice rather than what they can discriminate" (italics added). We tried to capture this "noticing" quality of awareness with the stimulus that participants were asked to detect, "an object". The subjective quality of awareness was assessed objectively by giving participants a clear, standardised definition of this detection stimulus (Wiens, 2006). Pilot testing showed that participants were more likely to have different response criteria when they were asked if either "a spider or a flower" was presented on each trial, and tended to share response criteria if they were instead asked if "an object" was presented (because the definition of "an object" - see procedure belowwas clearer than definitions of two specific objects, reducing the working memory requirements for the detection task). Using an "object" as the detection stimulus meant participants were asked to make different discriminations in the detection and identification tasks. However, because these tasks were administered concurrently and the detection task was always first, if responses to the identification task primed subjects on the ensuing detection trials, their bias would likely be

COGNITION AND EMOTION, 201327 (7) 1155 
to detect a spider or a flower-in which case the same stimulus discriminations would likely occur on the two tasks. To ensure that stimulus detection was measured under optimal conditions, the detection task was always first (Merikle \& Reingold, 1990). If participants fail to detect an object, and in turn discriminate a phobic from a non-phobic one, it may suggest that such discrimination occurred without awareness.

The subjective quality of awareness (detecting an "object") was also assessed objectively by using signal detection (SD) methods. Measures of awareness that rely on perceptual thresholds are often suboptimal because they don't tend to separate detection ability from response biases. If phobic participants detect threatening stimuli more often than others, it may simply reflect a tendency to report that they are presented more often. Critically, SD methods assess stimulus detection by separating detection of a target stimulus (hits) from this response bias. To measure response bias, control stimuli were created from the target images that had the same colours, luminance and general texture of the target images, but lacked any of their discernible features (Öhman \& Soares, 1993, 1994).

The control stimuli were also used to measure response bias in the second, perception index of the dissociation paradigm, a forced-choice identification task. For example, if there are two targets (spiders and flowers), chance-level performance is not necessarily $50 \%$ because participants may tend to respond with one category more than the other, especially if one is personally relevant (i.e., spiders for spider-phobic individuals). Thus, target identification must be directly compared to identification biases, the tendency to identify control stimuli as each of the target alternatives. Discrimination tasks become very difficult when the participant is asked to choose among four target categories (Green, Weber, \& Duncan, 1977), which may exceed the capacity of visual working memory (Baddeley, 1995). Thus, failure to discriminate may indicate lack of motivation rather than perceptual processing. We used two target categories in light of this potential problem. Incorrect identification of the alternative category (e.g., a flower is identified as a spider) provided a "false alarm" rate and thus secondary measure of response bias.

\section{METHOD}

\section{Participants}

In what they thought was an independent study, the Fear of Spiders Questionnaire (FSQ; Szymanski \& O'Donohue, 1995) was administered to 450 undergraduates at a public northeastern college. Those who scored in the top 15\% (most fearful) and bottom 50\% (not fearful) of the distribution of FSQ scores were contacted to participate. To validate these self-reports, participants engaged in a Behavioural Avoidance Test (BAT) with a live tarantula (see appendix). Those who identified themselves as spider-fearful but did not avoid the tarantula, and identified themselves as non-fearful but did avoid the tarantula, were excluded ( $10 \%$ of participants). As is typical of studies based on the dissociation paradigm (e.g., Haase et al., 1999), participants who generated insufficient undetected trials were excluded ( $<33 \%$ or eight target trials; $13 \%$ of participants). These screenings resulted in samples of 25 spiderphobic and 22 non-phobic participants, comparable in age and gender (total of 36 females and 11 males). Average age was 19.3 years $(S D=1.6)$.

\section{Design and overview}

Participants were presented with 24 masked images each of spiders and flowers and an equal number (48) of masked control stimuli (scrambled collages of the target images, described below) in a random sequence $(\mathrm{SOA}=25 \mathrm{~ms}$; duration of masking stimulus $=125 \mathrm{~ms}) .{ }^{1}$ After each masked target or control stimulus was presented, participants gave a detection response, followed by an

\footnotetext{
${ }^{1}$ The SOA is primarily a function of the refresh rate of the monitor, or how quickly it regenerates stimuli on the screen. By default, SuperLab erases the target stimulus before the masking stimulus is presented on the next refresh cycle. This makes the SOA between target and mask approximately $25 \mathrm{~ms}$ (SuperLab, personal communication).
} 
identification response. Thus, the variables of the experiment were Group (phobic or non-phobic; between-subjects) and Stimulus Type (spider, flower, and control; within-subjects), yielding a $2 \times 3$ factorial design with repeated measures. As described below, the detection and identification responses were used to measure dependent variables that were the basis of their respective analyses. Detection responses were classified as hits (either target is presented and detected), misses (either target is presented and not detected) or false alarms (inaccurate detection of target when a control stimulus is presented). With three types of stimuli, there were three identification measures: correct identification (e.g., a spider stimulus is identified as a spider), incorrect identification (a spider is identified as a flower, or a flower as a spider), and control identification (identification of control stimuli as either spiders or flowers).

\section{Measures, materials and equipment}

The Fear of Spiders Questionnaire (FSQ; Szymanski \& O'Donohue, 1995) is widely used to identify individuals who are relatively high or low in fear of spiders. Filler/unscored questions that disguised the intent of the questionnaire concerned fear of heights, snakes and needles.

A Behavioural Avoidance Test (BAT), an 11-point scale that involved approaching a live tarantula in a 10-gallon tank, was used to confirm participants' FSQ scores. Participants were asked to complete a series of tasks, one at a time, which brought them gradually closer to the tarantula. See the appendix for these tasks.

The target stimuli were colour images of spiders and flowers. In preliminary work, 25 of each were downloaded from entomology and botany department websites, and adjusted to fit within a window of $500 \times 500$ pixels. The flowers were members of either Brassia 'Rex Sakata', the "spider orchids", or of the genus Cleome, the "spider flowers". Like spiders, they have a central body (ovary/style) with multiple long, thin radiations (petals). The number of pixels, mean luminance, and mean contrast of luminance of the spider and flower images did not differ.
Following the procedure created by Öhman and Soares (1993), the control stimuli were created from a collage $(500 \times 500$ pixels $)$ of the spider and flower images. The images were cut into squares of $10 \times 10$ pixels, and 2,500 $(50 \times 50)$ squares were randomly chosen and shuffled so that the features of the target images were not discernible in the collage. The positions of the squares were randomly reshuffled to generate 50 control stimuli. As in prior studies that used this shuffling procedure (Öhman \& Soares, 1993, 1994; Wiens, Peira, Golkar, \& Öhman, 2008), the same rather than different control stimuli were created for the spider and flower images, i.e., there was one rather than two types of control stimuli so as to prevent a potential confound. (Spiderphobics may be more likely to mistake control stimuli created from the spider images for spiders, and may not similarly mistaken flower control stimuli for flowers.) The masking stimulus was an array of the repeating capital letters $A B C D$, $520 \times 520$ pixels. It totally covered the area where the target images appeared. The rationale for selecting it is presented below.

The stimuli were presented by SuperLab 4.0.1 on a Dell desktop PC with a19-inch LCD monitor. Screen resolution was $1024 \times 768$ pixels. Refresh rate was $60 \mathrm{~Hz}$. Stimuli were viewed at a distance of about $65 \mathrm{~cm}$, and within horizontal and vertical visual angles of 8.6 degrees.

\section{Procedure}

Participants were tested individually. They were instructed to focus on the $\mathrm{X}$ (fixation point) that would appear in the middle of the screen, which would be followed by a flash (target or control stimulus). Their first task was to indicate whether or not "an object" had been presented in the flash. They were given this definition: "By an object, I mean a physical object, any physical entity or thing that has a form" (www.dictionary.com). The participant was asked if she or he understood the definition, and it was carefully repeated until she or he understood that she or he was being asked if any object at all appeared in the flash. Following Becker and Rinck's (2004) recommendation to

COGNITION AND EMOTION, 201327 (7) 
avoid conservative response bias (e.g., responding "No" on each trial, which would preclude testing the hypothesis), the participant was told that half the time an object would be presented, half the time it wouldn't be, that this would vary randomly, so she or he should be mindful of that when guessing. Participants first did 15 detection practice trials. Then they were told a second task was being added: after indicating if an object was presented, they would indicate whether a spider or a flower had been presented, whether or not they previously reported that an object had been presented. Participants were told that when an object was presented, the two target categories would be shown equally often and that this would vary randomly, again to avoid excessive response bias (e.g., spider-phobics responding "Spider" on every identification trial). Participants were told that because this was a difficult task, there might be trials in which one of these stimuli was presented even though it seemed like neither of them was presented. Regardless, they should attend to all of the flashes, first indicate whether or not an object was presented, and then identify each flash as either a spider or a flower-even if their first response was that no object had been presented. Again, if they were unsure about whether a spider or flower was presented, they should give their best guess. These instructions were carefully explained to the participant, and were repeated when she or he read them on the screen. The participants then did a second set of 15 detection-identification practice trials to familiarise them with the concurrent tasks.

The actual task consisted of 96 detectionidentification trials, 24 each of the spider and flower pictures and 48 control stimuli presented in a random order. Each practice and actual trial began with the large $X$ appearing in the centre of the screen for two seconds, followed by a flash, which was either a target or control stimulus in the same location for $25 \mathrm{~ms}$, immediately followed by the ABCD masking stimulus for $125 \mathrm{~ms}$. After each presentation, a response screen appeared, asking whether or not an object was just presented. Participants clicked on one of these options with the left mouse button. Then a second response screen appeared, asking if a spider or a flower had been presented. Participants clicked on one of these options to indicate their identification response. Both response screens appeared until the participant responded (i.e., there was no time limit). Participants were instructed to respond as quickly as possible in order to gauge automatic responses. A blank screen appeared for an intertrial interval of one second. Each spider and flower image was presented once. The entire task took about 8.5 minutes.

\section{RESULTS}

\section{Data reduction and analysis: Stimulus detection}

Signal detection analyses (Macmillan \& Creelman, 2005) were used to assess detection of each target stimulus relative to the control stimuli. Crucially, such analyses separate stimulus detection $\left(d^{\prime}\right)$ from response bias $(C)$. The detection index $d^{\prime}=z$-hit rate $-z$-false alarm rate. Participants who can detect spiders would give a positive rating to more of the spider pictures (i.e., hit rate is high) than control stimuli (i.e., false alarm rate is low). The Objective Detection Threshold (ODT), where $d^{\prime}=0$, indicates that participants are equally likely to report the presence of a target stimulus on both target and control trials, and thus can't distinguish between them. The criterion $C$ is computed as $C=-0.5 \times(z$-hit rate $+z$-false alarm rate). Participants are considered unbiased if they are as likely to misinterpret noise as signal (false alarm) as they are likely to report signal as noise (miss).Thus, if the false alarm rate equals the miss rate, then $C=0$.

Detection performance of the phobic and nonphobic groups for the target stimuli is shown in Table 1 . The groups reported a relatively equal number of detection hits and misses for target and control trials (i.e., sufficient to test the hypothesis). The $d^{\prime}$ and $C$ values were statistically evaluated in 2 (Group: phobic or non-phobic) $\times$ 2 (Target Stimulus: spider or flower) analyses of variance (ANOVAs). For the ANOVA of $d^{\prime}$ values, neither the main effect of Group, 
$F(1,45)=1.14, p=.29$, nor the interaction of Group and Target $(F<1, n s)$ was significant. The main effect of Target Stimulus approached significance, $F(1,45)=3.70, p=.06$. A paired samples $t$-test showed that across groups, detection of flowers nearly exceeded that of spiders, $t(46)=$ $1.95, p=.058$. Interestingly, all $d^{\prime}$ means differed from 0 , showing that targets were distinguished from control stimuli-except the spider $d^{\prime}$ of the phobic group: one-sample $t(24)=1.25, p=.22$. Phobic flower $d^{\prime}$ : $t(24)=2.08, \quad p=.048$; Non-phobic spider $d^{\prime}: t(22)=2.29, p=.033$; Non-phobic flower $d^{\prime}: t(21)=3.27, p=.004$. That is, the phobic group could not distinguish between masked spiders and control stimuli. This result is consistent with the three studies cited in the introduction that did not find a phobic stimulus detection bias. Flower detection by both groups and spider detection by non-phobics are consistent with the $d^{\prime}$ values reported by Wiens et al. (2008) at about the same SOA (24 ms).

For the ANOVA of $C$ values, neither the main effect of Group, $F(1,45)=1.52, p=.22$, nor the interaction of Group and Target $(F<1, n s)$ was significant. The main effect of Target Stimulus approached significance, $F(1,45)=3.70, p=.06$. A paired samples $t$-test showed that across groups, $C$ for spiders nearly exceeded that for flowers, $t(46)=1.95, p=.058$, essentially showing that participants were more likely to respond to spiders than to control stimuli. For the phobic group, $C$ values for both spiders and flowers differed from $0, t(24)=3.40, p=.002$, and $t(24)=2.84$, $p=.009$, respectively. For the non-phobic group,
$C$ values for neither spiders nor flowers differed from $0, t(21)=1.75, p=.095$ and $t(21)=1.19$, $p=.25$, respectively. Thus, phobics were more likely than non-phobics to respond to both targets than to control stimuli.

\section{Data reduction and analysis: Spider-flower identification}

In the perception dissociation paradigm, detection-identification dissociation is assessed by analysing identification of detection hits and misses separately: those trials on which either a spider or a flower was presented and detected (hits), and those on which either was presented but not detected (misses; e.g., Haase et al., 1999; Merikle \& Reingold, 1990). The identification rate for each target category was the proportion of detection trials (hits or misses) in which a target stimulus was correctly identified. For example, this rate for spider misses would be the proportion of trials in which a spider was presented, not detected, and correctly identified as a spider. Each participant's readiness to respond with a target category (spider or flower) was the proportion of control stimuli that each category used. Even though the control stimuli couldn't be sorted into actual detection hits and misses (no object was presented), this response bias was also calculated separately for control trials on which a participant reported she or he detected and did not detect an object-control "hits" and "misses", respectively. The incorrect identification rate for each target category was the proportion of trials in which target spiders were identified as flowers, and

Table 1. Detection performance of the phobic and non-phobic groups

\begin{tabular}{|c|c|c|c|c|c|}
\hline Group & Stimulus & Hits & $F A$ & $d^{\prime}$ & $C$ \\
\hline \multicolumn{6}{|l|}{ Phobic } \\
\hline & Spiders & $0.372(0.04)$ & $0.331(0.04)$ & $0.113(0.09)$ & $0.429(0.17)$ \\
\hline & Flowers & $0.418(0.05)$ & $0.331(0.04)$ & $0.227(0.10)$ & $0.384(0.17)$ \\
\hline \multicolumn{6}{|c|}{ Non-phobic } \\
\hline & Spiders & $0.471(0.04)$ & $0.400(0.05)$ & $0.263(0.11)$ & $0.260(0.14)$ \\
\hline & Flowers & $0.518(0.05)$ & $0.400(0.05)$ & $0.376(0.11)$ & $0.203(0.17)$ \\
\hline
\end{tabular}

Notes: $\mathrm{FA}=$ false alarm rate; $d^{\prime}=$ perceptual sensitivity; $C=$ criterion or response bias. $d^{\prime}=z(\mathrm{H})-z(\mathrm{FA}) ; C=-0.5 \times[z(\mathrm{H})+z(\mathrm{FA})]$. Values outside of the parentheses represent mean scores of the detection measures. Values within parentheses represent standard error of measurement. 
Table 2. Identification performance of the phobic and non-phobic groups contingent on detection hits and misses

\begin{tabular}{|c|c|c|c|c|c|c|c|}
\hline \multirow[b]{3}{*}{ Group } & \multirow[b]{3}{*}{ Target stimulus } & \multicolumn{6}{|c|}{ Detection } \\
\hline & & \multicolumn{3}{|c|}{ Misses } & \multicolumn{3}{|c|}{ Hits } \\
\hline & & Correct (\%) & Control & Incorrect (\%) & Correct (\%) & Control & Incorrect (\%) \\
\hline \multicolumn{8}{|l|}{ Phobic } \\
\hline & Spiders & 0.620 & 0.454 & 0.525 & 0.574 & 0.421 & 0.400 \\
\hline & Flowers & 0.475 & 0.546 & 0.380 & 0.574 & 0.580 & 0.426 \\
\hline \multicolumn{8}{|c|}{ Non-phobic } \\
\hline & Spiders & 0.619 & 0.618 & 0.605 & 0.485 & 0.395 & 0.397 \\
\hline & Flowers & 0.395 & 0.382 & 0.381 & 0.604 & 0.606 & 0.515 \\
\hline
\end{tabular}

Notes: Correct $(\%)=$ proportion of target trials correctly identified. Control = proportion of control trials identified as a target stimulus. Incorrect $(\%)=1-$ Correct $(\%)$ of the alternative target stimulus, e.g., the proportion of spider misses that were identified as flowers. Values represent mean scores.

target flowers were identified as spiders. This is a false alarm rate and thus a secondary measure of response bias (Öhman \& Soares, 1993, 1994).

Table 2 shows these three measures for each group with respect to each target stimulus, contingent on detection hits and misses. These data are also depicted graphically for the phobic group in Figure 1, and for the non-phobic group in Figure 2. In order to assess identification of the target stimuli by the groups relative to response biases, the three measures were statistically evaluated in 2 (Group: phobic or non-phobic) $\times 2$ (Target Stimulus: spider or flower) $\times 3$ (Measure: correct, incorrect, and control identification rate) ANOVAs. Again, these ANOVAs were conducted separately for detection hits and misses.

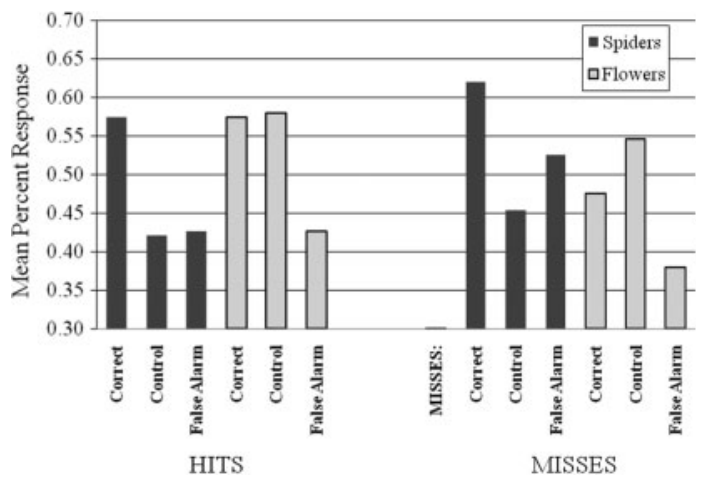

Figure 1. Correct identification, control and false alarm rates of the phobic group contingent on detection hits and misses.
Recall the hypothesis was that a stimulus detection-identification would be specific to phobic stimuli: the phobic group would identify spiders better than chance whether or not they detect them (i.e., on both detection hits and misses), while the non-phobic group would only identify spiders when they detect them (i.e., on detection hits only). That is, we predicted a threeway interaction between Group, Target Stimulus and Measure for detection misses, and a two-way interaction between Target Stimulus and Measure for detection hits. As three-way ANOVAs involve a variety of interactions between factors, only these specific interactions and relevant follow-up analyses are presented in order to clarify hypothesis testing.

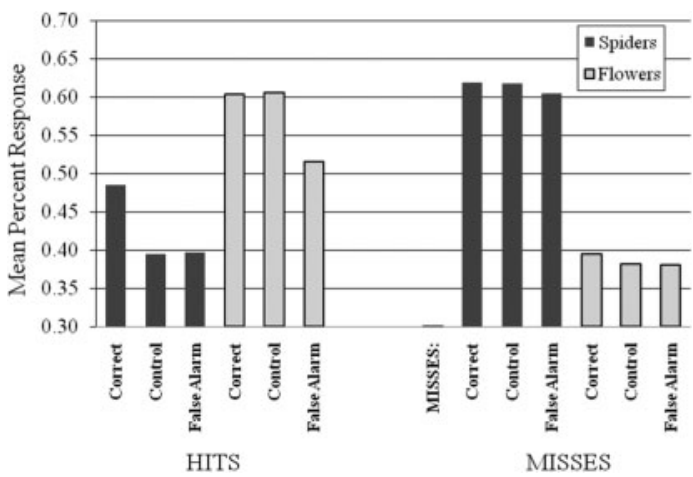

Figure 2. Correct identification, control and false alarm rates of the non-phobic group contingent on detection hits and misses. 
Detection misses: Spider-flower identification. In the $2 \times 2 \times 3$ ANOVA of detection misses, the Group $\times$ Target Stimulus $\times$ Measure interaction effect was significant, $F(2,90)=4.70, p=.036$. As can be seen from the means in Table 2, this interaction appears to stem from identification of spider misses by the phobic group. This was confirmed by $2 \times 3$, Group $\times$ Measure ANOVAs, each conducted separately for spiders and flowers. For spider misses, the Group $\times$ Measure interaction effect was significant, $F(2,90)=3.21, p=$ .047 , showing that spider identifications differed as a function of group. For flower misses, this interaction was not significant, $F(2,90)=1.60$, $p=.213$. Further, a one-way ANOVA of the phobic group's spider identification measures was highly significant, $F(2,48)=6.58, p=.004$. A Tukey's test showed that correct identification of undetected spiders exceeded identification of control stimuli as spiders $(M D=0.165, p=.01$, effect size $d=0.78$ ). The same comparison with the second measure of response bias - incorrect identification of flowers as spiders - was nearly significant $(M D=0.095, p=.065)$. A paired samples $t$-test of this comparison was significant, $t(24)=2.27, p=.032$ (effect size $d=0.48$ ). There were no significant effects for flowers or for the non-phobic group.

These findings show that the phobic group identified undetected spiders (i.e., misses) better than chance. Identification of spider misses did not reflect the influence of a handful of phobic participants, but most of them. It exceeded both measures of response bias for 13 of 24 phobics, and exceeded at least one of these measures for 22 of them. By comparison, identification of undetected flowers exceeded both measures of response bias for only five phobics, and exceeded at least one of these measures for 15 of them. However, phobic participants who had conservative detection biases-i.e., who tend to respond that they did not detect an object, and hence have a high number of misses - but are very good at discriminating spiders from flowers may have been more likely to identify undetected spiders. To address this potential confound, correct identification of undetected spiders by the phobic group was correlated with response criterion $(C)$ on the detection task. This correlation did not approach significance, $r(25)=-.26, p=.21$.

Detection hits: Spider-flower identification. Recall that the hypothesis for detection hits was a twoway interaction between Target Stimulus and Measure: both the phobic and non-phobic groups would identify spider hits better than chance. In the $2 \times 2 \times 3$ ANOVA of detection hits, the Group $\times$ Target Stimulus $\times$ Measure interaction effect was not significant $(F<1, n s)$, showing that identification of detected target stimuli did not differ between groups. Across groups, the main effect of Target Stimulus $(F(2,90)=11.2$, $p=.002)$, the main effect of Measure $(F(2,90)=$ 9.34, $p=.0001)$, and the interaction effect of Target Stimulus and Measure $(F(2,90)=4.01$, $p=.022)$ were all significant. The latter effect showed that identification performance varied as a function of target stimulus. As shown by the pattern of the means in Figure 2, this interaction appears to stem from enhanced identification of detected spiders. Pairwise comparisons showed that both groups identified spiders if they previously detected an object, relative to both measures of response bias. For phobics, the results of Tukey's tests were: $p=.01$ for control comparison, effect size $d=0.64 ; p=.007$ for incorrect identification comparison, $d=0.74$. For non-phobics, the results of paired-samples $t$-tests were: $t(21)=$ 2.00, $p=.05, d=0.44$, for both comparisons. Both groups tended to identify flowers relative to one of the response bias measures - incorrect identification of spiders as flowers. For phobics: Tukey's $p=.01$, effect size $d=0.64$. For nonphobics: paired samples $t(21)=1.95, p=.06, d=$ 0.45 . This pattern of results shows that across groups, detected target stimuli were identified better than chance, though this effect was stronger for spiders than for flowers.

\section{DISCUSSION}

The results indicate that the phobic group correctly identified both detected and undetected spiders-hits and misses-better than chance,

COGNITION AND EMOTION, 201327 (7) 
relative to both measures of response bias (identification of control stimuli as spiders, and incorrect identification of flowers). However, they did not exhibit the same dissociation between detection and identification of flower$s$ - that is, they did not identify undetected flowers (misses) better than chance. The nonphobic group did not exhibit detection-identification dissociation for either spiders or flowers. This pattern of findings shows that dissociation between stimulus detection and identification was specific to phobic stimuli. That is, the results indicate a phobic object can be distinguished from a fear-irrelevant one in the absence of detection of an object. As noted above, this dissociation did not reflect a handful of phobic participants, but most of them.

These results are consistent with the body of research referred to at the outset attesting to dissociations between awareness of and responses to phobic stimuli in particular. Of particular note is the work of Öhman and colleagues, who demonstrated that autonomic responses can be elicited by and conditioned to masked phobic and fear-relevant objects, respectively(Esteves et al., 1994; Katkin et al., 2001; Öhman \& Soares, 1993, 1994, 1998). The current findings similarly suggest that phobic processing is mediated by mechanisms for specific threatindicating features. Recall that most of the flower images were either "spider orchids" or "spider flowers", which like spiders have a central body with multiple, long, thin radiations. Nonetheless, phobic group distinguished spiders from them on detection misses. Thus, the current findings build on the work of Öhman and colleagues by showing that the recognition of phobic stimuli can itself be dissociated from awareness. In this sense, they provide a missing link between stimulus and response in masked phobic phenomena.

The current findings are also consistent with signal detection studies that found that phobic individuals were no more likely to detect their masked feared stimulus than non-phobic participants (see introduction for citations). Enhanced recognition of masked phobic stimuli emerged not in the detection task, but in the identification of undetected stimuli. Previously, a perceptual bias for phobic stimuli only emerged when threatening stimuli vied for attention with non-threatening stimuli (see Mogg \& Bradley, 1998; Williams et al., 1997, for reviews). The current findings suggest that a phobic object is also preferentially perceived when it is individually presented - if not only detection but also identification of the stimuli is concurrently assessed.

The results are also consistent with prior findings of detection-identification dissociations for verbal and simple non-verbal stimuli (Benzschawel \& Cohn, 1985; Haase et al., 1999; Lindner, 1968; Marcel, 1983; Merikle \& Reingold, 1990; Rollman \& Nachmias, 1972). The findings extend such dissociations to the new category of emotional stimuli, and suggest that they follow a different boundary condition than verbal stimuli. Recall that on the basis of their finding of recognition of undetected words and not non-words, Merikle and Reingold (1990) concluded that detection-identification dissociations would be evidenced by familiar stimuli, i.e., those that are represented in memory. In this study, stimulus familiarity was not the factor that determined such dissociations. Rather, the affective and personal relevance of the stimuli determined if detection-identification dissociation occurred. The effect size of this dissociation for phobic stimuli was comparable to that for verbal stimuli (Merikle \& Reingold, 1990).

This study had a few limitations. An LCD screen was used to present the stimuli, which may not be as reliable in providing very brief stimulus durations as other screens (Wiens et al., 2004). Another limitation was a lower number of trials than is typical of the dissociation paradigm. Phobic stimuli are personally relevant and emotionally charged. Discrimination tasks typically employ no more than 20-24 trials per stimulus category, and 6-10 trials at a very brief SOA, in order to prevent awareness from developing (Öhman \& Soares, 1993, 1994; Wiens et al., 2008). This study had about four times more 
target trials than these studies, and larger phobic and non-phobic samples than these studies so that the total number of trials for the phobic group $(2,400)$ was considerable. Nonetheless, the lower number of trials than is typical of the perception dissociation paradigm may have limited the sensitivity of the analysis. Another limitation of this study was that participants were asked to make different discriminations on the detection and identification tasks ("an object" vs. "a spider or a flower", respectively). However, as noted in the introduction, because the stimulus detection and identification tasks were administered concurrently, if participants were primed by their responses to identification trials on the ensuing detection trials, they were likely biased to detect a spider or a flower (and would thus be making the same discriminations on the two tasks). This was confirmed by a funnelled interview administered immediately after the experiment, on which no one reported that they were looking for any other object. Regardless, the possibility that the detection and identification tasks may have captured different kinds of conscious information - that the detection measure was not an exhaustive measure of conscious awareness - cannot be ruled out.

\section{What does the detection-identification dissociation for phobic stimuli mean?}

Signal detection theory (SDT) predicts that identification of detection misses will occur because the response criteria associated with the detection and identification tasks are assumed to vary independently, especially if there are task differences (see Macmillan, 1986). As it makes no reference to awareness, there is no straightforward SDT interpretation of detection-identification dissociations:

... there is no point in the (SDT) model where one can distinguish between conscious and unconscious perception...the question remains as to whether correct identification was based on unconscious perceptual processes, or perhaps a vague awareness that the target + noise field was more similar to one target than another. (Haase et al., 1999, p. 991)
In this study, is there a valid basis for inferring that identification of undetected target trials refers to conscious or unconscious processes? As noted in the introduction, validation of a stimulus detection measure relies on the demonstration of qualitative differences associated with the presumed "aware" and "unaware" states assessed by the measure (Merikle \& Reingold, 1990; Wiens, 2006). This study yielded ample evidence of qualitative differences associated with detection hits and misses. Confirming the hypotheses, phobic identified spiders better than chance on detection misses as well as hits, while non-phobics only identified spiders better than chance on detection hits. Further, phobics did not show the same detection-identification dissociation for flowers. They only identified flowers better than chance on detection hits, not misses. That is, the detection measure was associated with qualitative differences in perception between a phobic and a non-phobic group, and between a phobic and a non-phobic stimulus. Interestingly, the phobic group identified spider misses better than chance despite the fact that spider detection $\left(d^{\prime}\right)$ did not differ from the ODT $\left(d^{\prime}=0\right)$. As developed below, these differences presumably demonstrated a learned emotional mechanism.

Identification performance across groups also yielded qualitative differences between detection hits and misses. Across groups, spiders were identified better than chance on detection hits, but not misses. Flowers were similarly identified better than chance on detection hits (relative to one of the response bias measures), but not on detection misses. Thus, generally speaking, the target stimuli tended to be identified when an object was previously detected, as would be expected if detection hits and misses reflected a variation in their awareness of the target stimuli. Further, as demonstrated by the interaction effect of Target Stimulus and Measure, enhanced recognition of spiders emerged on detection hits, but not misses. That is, across groups, spiders were more likely to be identified than flowers when an object was previously detected. This may have reflected a biological readiness to identify detectable, fear-relevant 
stimuli. ${ }^{2}$ The most parsimonious interpretation of these various differences in perception associated with detection hits and misses is that they were based on awareness of the stimuli. For example, the alternative interpretation that the detection-identification dissociation for phobic stimuli stemmed from task differences must explain how such differences interacted with the affective significance of the stimuli and the participant variable to generate the dissociation. That requires the interpretation to become less parsimonious than one that the dissociation stemmed from awareness of the stimuli (Merikle \& Reingold, 1990).

Alternatively, it could be argued that the participants were not unaware of the target stimuli because three of the four detection $\left(d^{\prime}\right)$ values for the target stimuli differed from 0 (see Data reduction and analysis: Stimulus detection). Thus, conclusions about unaware processes cannot be drawn. However, in studies based on the DID paradigm, $d^{\prime}$ for the target stimuli typically significantly differ from 0 (Benzschawel \& Cohn, 1985; Green et al., 1977; Haase et al., 1999). In the classic DID study described in the introduction-Merikle and Reingold's (1990) analysis of lexical decision - $d^{\prime}$ rates were much higher than 0 (ranging from 1.35 to 2.03). That is because in DID studies $d^{\prime}$ is not used to show that participants are unaware of the target stimuli, but that they were able to distinguish a detection stimulus (in this study, an "object") from noise/ blanks. If participants can't detect the presence of the target stimuli $\left(d^{\prime}=0\right)$, then there will not be adequate detection hits. Thus, it will not be possible to test the DID hypothesis by comparing identification of the target stimuli on detection hits and misses. The $d^{\prime}$ values refer to target stimuli that were detected (hits) and control stimuli that were incorrectly detected (false alarms). They do not refer to undetected stimuli-misses. In the DID paradigm, conclusions about unaware processes regard misses. Thus, $d^{\prime}$ values are less relevant to making claims about unaware processes. As described in the introduction, the DID paradigm circumvents the use of an adequate awareness threshold $\left(d^{\prime}=0\right)$ which researchers have not been able to agree on-by showing that detection hits and misses are associated with qualitative differences in perception, as described above.

Regardless, recognition of a phobic object in the absence of detection of an object does not necessarily demonstrate that the former was unconsciously perceived. Rather, the findings suggest that the detection task was a valid measure of awareness, and are consistent with the interpretation that the phobic stimuli were so perceived. Detection-identification dissociations raise fundamental questions about processing. If "perception" refers to not only the organisation but also the interpretation of stimuli by the occipital-temporal cortex-i.e., a thoroughly cognitive process - then the phrase "unconscious perception" is likely a contradiction in terms, and any debate about the meaning of detectionidentification dissociations ends by fiat. If the findings do not show that the phobic group perceived a threatening stimulus without awareness, they at least show that this group apprehended its emotional significance.

\footnotetext{
${ }^{2}$ The response bias measures also yielded qualitative differences associated with hits and misses. On detection misses, false alarm rates for spiders exceeded those for flowers, indicating that missed flowers were more likely to be interpreted as spiders than vice versa. Paired samples $t$-tests showed that this effect was exhibited by both phobics, $t(24)=2.14, p=.043$, and by non-phobics, $t(21)=3.07, p=.006$. On detection hits, however, these false alarm rates were comparable in both groups. That is, non-phobic/ fear-irrelevant stimuli were misinterpreted as phobic/fear-relevant only when either group did not detect the former stimuli. Furthermore, a paired $t$-test showed that while non-phobics more likely to interpret "undetected" control stimuli as spiders rather than flowers, they were more likely to interpret "detected" control trials as flowers rather than as spiders, $t(21)=2.79, p=.011$, in both cases. This suggests that when the perceptual situation was utterly ambiguous, they may have used different interpretation strategies based on their awareness of the stimuli, as was reported by a few non-phobics during debriefing, e.g., "If I couldn't make it (the stimulus) out, I thought it was probably something interesting. If I could make it out, it was probably something ordinary."
} 


\section{Theoretical implications and future studies}

A dissociation between detection and identification of phobic stimuli is consistent with a number of theories that propose an unconscious processing system for threatening stimuli. LeDoux (1996) discovered a direct pathway from the thalamus to the amygdala for conditioning fear responses in rats, what he called the "low-road". Morris et al. $(1998,1999)$ demonstrated that a similar subcortical circuit appears to be operating in the human brain (as did a number of subsequent neuroimaging studies, e.g., Carlsson et al., 2004; Phelps, 2005; Whalen et al., 2004; Williams et al., 2004). LeDoux (1996) proposed that this subcortical circuit functions as an unconscious memory system for threat-indicating features in order to initiate autonomic responses much faster than can be accomplished by a longer cortical circuit (the "high-road"). As noted in the introduction, Öhman and Mineka (2001) proposed the related idea of a fear module, an encapsulated neural system for automatically processing stimuli that posed threats in our evolutionary history.

How might an unconscious system for threatening stimuli generate dissociation between detection and identification of them? Backwards masking is believed to short-circuit conscious cognition so that unconscious mechanisms presumably mediate perception (Enns \& Di Lollo, 2000; Öhman \& Mineka, 2001). In the current study, masking may have prevented conscious detection of stimuli without preventing critical phobic features from being apprehended by an unconscious system. These features would be fed up to a conscious, cognitive system, in turn, enhancing discrimination between phobic and non-phobic stimuli. This theory could be tested by coupling the stimulus detection-identification paradigm of this study with fMRI. If the results are replicated and detection-identification dissociation for phobic stimuli is associated with a distinct pattern of neural activation-different than that associated with detection-identification consistency, or recognition of detected stimuli-it would validate the theory.
Future studies of detection-identification dissociations for phobic stimuli should assess doseresponse relationships (e.g., Wiens et al., 2008). Rather than bifurcating awareness into "aware/ unaware" states, dose-response relationships assume that awareness is on a continuum. For example, SOA would be manipulated in order to compare detection and identification of phobic stimuli at different levels of awareness. Rather than relying on correlational demonstrations of qualitative differences in perception at the same SOA, manipulating SOA, and thus level of awareness of the target stimuli, would provide a stricter test of detection-identification dissociation for phobic stimuli. The dissociation would be expected to emerge at specific SOAs/levels of awareness, bounded by upper and lower thresholds. Such boundaries would further suggest non-conscious perceptual mechanisms for phobic stimuli.

\section{REFERENCES}

Baddeley, A. D. (1995). Working memory. In M. Gazzaniga (Ed.), The cognitive neurosciences (pp. 754-764). Cambridge, MA: Bradford/MIT Press.

Barlow, D. H. (2002). Anxiety and its disorders: The nature and treatment of anxiety and panic. New York, NY: Guilford Press.

Becker, E. S., \& Rinck, M. (2004). Sensitivity and response bias in fear of spiders. Cognition and Emotion, 18, 961-976. doi:10.1080/026999303 41000329

Benzschawel, T., \& Cohn, T. E. (1985). Detection and recognition of visual targets. Journal of the Optical Society of America A, 2, 1543-1550. doi:10.1364/ JOSAA.2.001543

Bowers, K. S. (1984). On being unconsciously influenced and informed. In K. S. Bowers \& D. Meichenbaum (Eds.), The unconscious reconsidered (pp. 227-272). New York, NY: Wiley.

COGNITION AND EMOTION, 201327 (7) 
Carlsson, K., Peterson, K. M., Lundqvist, D., Karlsson, A., Ingvar, M., \& Öhman, A. (2004). Fear and the amygdala: Manipulation of awareness generates differential cerebral responses to phobic and fearrelevant (but non-feared) stimuli. Emotion, 4, 340353. doi:10.1037/1528-3542.4.4.340

Enns, J. T., \& Di Lollo, V. (2000). What's new in visual masking? Trends in Cognitive Sciences, 4(9), 345-352. doi:10.1016/S1364-6613(00)01520-5

Esteves, F., Dimberg, U., \& Öhman, A. (1994). Automatically elicited fear: Conditioned skin conductance responses to masked facial expressions. Cognition and Emotion, 8, 393-413. doi:10.1080/ 02699939408408949

Eysenck, M. W. (1992). Anxiety: The cognitive perspective. Hove: Erlbaum.

Green, D. M., Weber, D. L., \& Duncan, J. E. (1977). Detection and recognition of pure tones in noise. Journal of the Acoustical Society of America, 62, 948954. doi:10.1121/1.381588

Haase, S. J., Theios, J., \& Jenison, R. (1999). A signal detection theory analysis of an unconscious perception effect. Perception \& Psychophysics, 61, 986-992. doi:10.3758/BF03206912

Holender, D., \& Duscherer, K. (2004). Unconscious perception: The need for a paradigm shift. Perception E Psychophysics, 66, 872-881. doi:10.3758/ BF03194980

Katkin, E. S., Wiens, S., \& Öhman, A. (2001). Nonconscious fear conditioning, visceral perception, and the development of gut feelings. Psychological Science, 12, 366-370. doi:10.1111/1467-9280.00368

LeDoux, J. E. (1996). The emotional brain. New York, NY: Simon \& Schuster.

Lindner, W. A. (1968). Recognition performance as a function of detection criterion in a simultaneous detection-recognition task. Journal of the Acoustical Society of America, 44, 204-211. doi:10.1121/ 1.1911056

Lovibond, P. F., \& Shanks, D. R. (2002). The role of awareness in Pavlovian conditioning: Empirical evidence and theoretical implications. Journal of Experimental Psychology and Animal Behaviour Processing, 28, 3-26.

Macmillan, N. A. (1986). The psychophysics of subliminal perception. Behavioral \& Brain Sciences, 9, 38-39. doi:10.1017/S0140525X00021427

Macmillan, N. A., \& Creelman, C. D. (1991). Detection theory: $A$ user's guide. New York, NY: Cambridge University Press.
Macmillan, N. A., \& Creelman, C. D. (2005). Detection theory: $A$ user's guide (2nd ed.). Mahwah, NJ: Erlbaum.

Marcel, A. J. (1983). Conscious and unconscious perception: Experiments on visual masking and word recognition. Cognitive Psychology, 15, 197237. doi:10.1016/0010-0285(83)90009-9

Mathews, A., \& Mackintosh, B. (1998). A cognitive model of selective processing in anxiety. Cognitive Therapy and Research, 22, 539-560. doi:10.1023/ A:1018738019346

Merikle, P. M. (1992). Perception without awareness: Critical issues. American Psychologist, 47, 792-795. doi:10.1037/0003-066X.47.6.792

Merikle, P. M., \& Daneman, M. (2000). Conscious vs. unconscious perception. In M. S. Gazzaniga (Ed.), The new cognitive neurosciences (2nd ed., pp. 12951303). Cambridge, MA: MIT Press.

Merikle, P. M., \& Reingold, E. M. (1990). Recognition and lexical decision without detection: Unconscious perception? Journal of Experimental Psychology: Human Perception E Performance, 16, 574-583. doi:10.1037/0096-1523.16.3.574

Mogg, K., \& Bradley, B. P. (1998). A cognitivemotivational analysis of anxiety. Behavior Research and Therapy, 36, 809-848. doi:10.1016/S00057967(98)00063-1

Morris, J. S., Öhman, A., \& Dolan, R. J. (1998). Modulation of human amygdala activity by emotional learning and conscious awareness. Nature, 383, 467-470. doi:10.1038/30976

Morris, J. S., Öhman, A., \& Dolan, R. J. (1999). A subcortical pathway to the right amygdala mediating "unseen" fear. Proceedings of the National Academy of Sciences, USA, 96, 1680-1685. doi:10.1073/ pnas. 96.4 .1680

Öhman, A., \& Mineka, S. (2001). Fears, phobias, and preparedness: Toward an evolved module of fear and fear learning. Psychological Review, 108, 483-522. doi:10.1037/0033-295X.108.3.483

Öhman, A., \& Soares, J. (1993). On the automatic nature of phobic fear: Conditioned electrodermal responses to masked fear-relevant stimuli. Journal of Abnormal Psychology, 102, 121-132. doi:10.1037/ 0021-843X.102.1.121

Öhman, A., \& Soares, J. (1994). "Unconscious anxiety": Phobic responses to masked stimuli. Journal of Abnormal Psychology, 103, 231-240. doi:10.1037/ 0021-843X.103.2.231

Öhman, A., \& Soares, J. (1998). Emotional conditioning to masked stimuli: Expectancies for aversive

\section{6}


outcomes following nonrecognized fear-relevant stimuli. Journal of Experimental Psychology: General, 127, 69-82. doi:10.1037/0096-3445.127.1.69

Pessoa, L., \& Adolphs, R. (2010). Emotion processing and the amygdala: From a "low road" to "many roads" of evaluating biological significance. Nature Reviews Neuroscience, 11, 773-780. doi:10.1038/ nrn2920

Phelps, E. A. (2005). The interaction of emotion and cognition: The relation between the human amygdala and cognitive awareness. In R. R. Hassin, J. S. Uleman \&J. A. Bargh (Eds.), The new unconscious (pp. 61-76). New York, NY: Oxford University Press.

Rollman, G. B., \& Nachmias, J. (1972). Simultaneous detection and recognition of chromatic flashes. Perception E Psychophysics, 12, 309-314. doi:10.375 8/BF03207211

Szymanski, J., \& O'Donohue, W. (1995). Fear of spiders questionnaire. Journal of Behavior Therapy and Experimental Psychiatry, 26, 31-34. doi:10.1016/0005-7916(94)00072-T

Weiskrantz, L., Warrington, E. K., Sanders, M. D., \& Marshall, J. (1974). Visual capacity in hemianopic field following a restricted occipital ablation. Brain, 97, 709-728. doi:10.1093/brain/97.1.709

Whalen, P. J., Kagan, J., Cook, R. G., Davis, F. C., Kim, H., Polis, S., .. Johnstone, T. (2004). Human amygdala responsivity to masked fearful eye whites. Science, 306, 2061. doi:10.1126/science. 1103617

Wiens, S. (2006). Current concerns in visual masking. Emotion, 6, 675-680. doi:10.1037/1528-3542.6.4.675

Wiens, S., Fransson, P., Dietrich, T., Lohmann, P., Ingvar, M., \& Öhman, A. (2004). Keeping it short: A comparison of methods for brief picture presentation. Psychological Science, 15, 282-285. doi:10.1111/ j.0956-7976.2004.00667.x

Wiens, S., Peira, N., Golkar, A., \& Öhman, A. (2008). Recognizing masked threat: Fear betrays but disgust you can trust. Emotion, 8, 810-819. doi:10.1037/ a0013731
Williams, J. M. G., Watts, F. N., MacLeod, C., \& Mathews, A. (1997). Cognitive psychology and emotional disorders (2nd ed.). Chichester: Wiley.

Williams, M. A., Morris, A. P., McGlone, F., Abbott, D. F., \& Mattingley, J. B. (2004). Amygdala responses to fearful and happy facial expressions under conditions of binocular suppression. Journal of Neuroscience, 24(12), 2898-2904. doi:10.1523/ JNEUROSCI.4977-03.2004

Windmann, S., \& Kruger, T. (1998). Subconscious detection of threat as reflected by an enhanced response bias. Consciousness and Cognition, 7, 603633. doi:10.1006/ccog.1998.0337

Winton, E. C., Clark, D. M., \& Edelmann, R. J. (1995). Social anxiety, fear of negative evaluation and the detection of negative emotion in others. Behavior Research and Therapy, 33, 193-196. doi:10.1016/0005-7967(94)E0019-F

\section{APPENDIX}

\section{The 11-point Behavioural Avoidance Test}

0 . Will not enter room

1. Enters the room but approaches no further

2. Comes within five feet of the tank but no further

3. Stands next to tank

4. Removes blanket covering tank

5. Places hands on two sides of tank closest to spider

6. Removes top cover of tank, and puts it back

7. Removes top cover of tank, puts face over open tank, and replaces cover

8. Removes top cover of tank, puts face over open tank and describes spider in detail

9. Removes top cover of tank, and places entire hand inside of tank horizontally

10. Reports willingness to touch spider (asked but never allowed) 